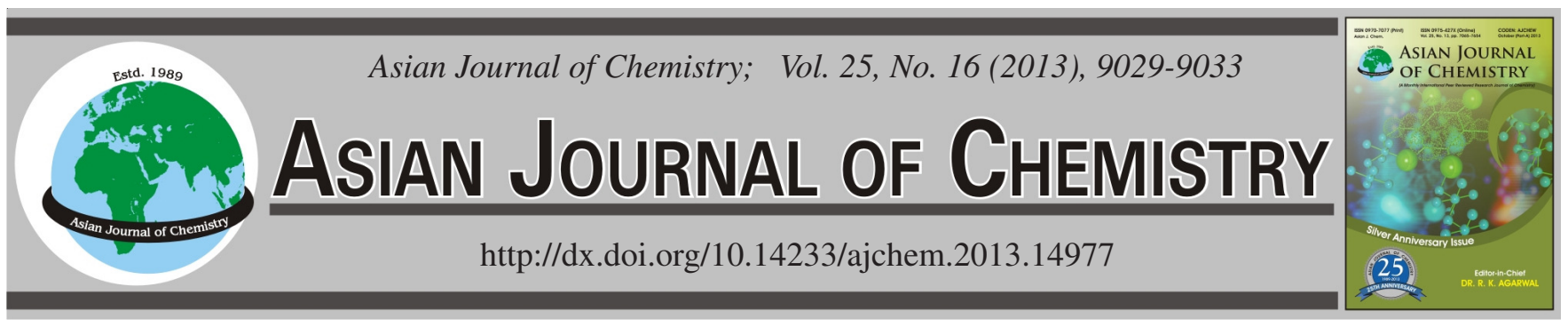

\title{
Simulated and Experimental Research on Increasing the Monomer Conversion of Emulsion Polymerization for Styrene Butadiene Rubber
}

\author{
YAN-JiAng JiN $^{1,2}$, BEN-XIAN SHEN ${ }^{1}$, LeI YANG ${ }^{2}$ and Ji-GANG ZhaO ${ }^{1, *}$
}

${ }^{1}$ State Key Laboratory of Chemical Engineering, East China University of Science and Technology, Shanghai 200237, P.R. China

${ }^{2}$ Jilin Petrochemical Company, PetroChina, Jilin 132021, P.R. China

*Corresponding author: Fax: +86 21 64252851; Tel: +86 21 64252916; E-mail: zjg@ecust.edu.cn

\begin{abstract}
The nucleation stage in emulsion polymerization for styrene-butadiene-rubber was established by classic theory and the influences of emulsifier and initiator concentration on the reaction were simulated by Monte Carlo method. The results showed that the relationship of the number of latex particle $[\mathrm{Np}]$, emulsifier concentration $[\mathrm{S}]$ and initiator concentration $[\mathrm{I}]$ is to $\mathrm{Np}=\mathrm{k}[\mathrm{S}]^{0.6062}[\mathrm{I}]^{0.4055}$, which agrees well with the Smith-Ewart theory. The simulation results showed that the monomer conversion in emulsion polymerization increased from 62 to $70 \%$ in the premise of ensuring product quality, the emulsifier incremental amount between 12.6 and $22.16 \%$ and the initiator incremental amount between 12.9 and $34.89 \%$. The experiments were investigated on increasing monomer conversion by emulsifier and emulsifier incremental amount in emulsion polymerization for styrene-butadiene-rubber according to the simulation results. The experimental results verify the simulation results.
\end{abstract}

Key Words: Monte Carlo simulation, Styrene butadiene rubber, Emulsion polymerization, Polymerization yield, Nucleation stage.

ᄂ - - - - - - - - - - - - - - - - - - - - - - - - - - - -

\section{INTRODUCTION}

As one of the most important polymers with wide application, copolymers of styrene and butadiene are generally synthesized using emulsion polymerization methods ${ }^{1}$. The performances of styrene-butadiene rubber are deteriorated by increasing the conversion. The final conversion in emulsion copolymerization of styrene and butadiene is usually limited to relatively low levels (e.g., about $60 \%$ ) for several decades in the industrial processes ${ }^{1}$. Increasing the monomer conversion can not only increase the utilization of monomer, reduce the recycling energy consumption, but also increase the capacity of the device. Therefore, Japan and other countries committed to study increasing the monomer conversion of styrene/butadiene rubber since $1960 \mathrm{~s}$ and made some progress such as the final monomer conversion has increased to 65 to $70 \%{ }^{2-4}$. A lot of experiments should do to increase the monomer conversion rate of monomer in the traditional way, as for dozens of substances in emulsion polymerization, which was cost a lot of money and time. So it is difficult to investigate on increasing the monomer conversion for emulsion polymerization by experimental at all ${ }^{5-9}$.

The emulsion co-polymerization process is a random process ${ }^{10-12}$, so it could be studied by Monte-Carlo simulation. In this work, the nucleation stage of emulsion polymerization of styrene was simulated with Monte Carlo method and the influences of the initial concentration of emulsifier and initiator on the reaction were simulated, which would provide the references of adjusting the recipe for increasing the monomer conversion. Finally, the simulation results invalid by doing some experiments.

EXPERIMENTAL
Monomers were styrene (analytical reagent, Shanghai
lingfeng chemical reagent Co. Ltd.) and butadiene (analytical
reagent, Shanghai Wetrycriterion Gas Co. Ltd.). Emulsifier is
potassium soap of disproportionated rosin (15.1\%). The compo-
sition of electrolyte solution is phosphoric acid, sodium
hydroxide, ethylene diamine tetraacetic acid tetra-sodium salt
and sodium methylenedinaphthalene disulphonate. Activator
solution is ferric sodium ethylene diamine tetraacetate and
sodium formaldehyde sulfoxylate. The modifier is tert-dodecyl
mercaptan [TCI (Shanghai) Development Co. Ltd.]. The short-
stop solution is sodium dimethyl dithiocarbamate, sodium
nitrite and sodium polysulfide. The oxidant is para-menthane
hydroperoxide. The unspecified chemicals were all come from
organic synthesis factory of Jilin pertrochemical company.
The nucleation stage in emulsion polymerization for
styrene-butadiene-rubber was established by Harkins and Smith-
Ewart theory. Some reasonable assumptions are as follows:




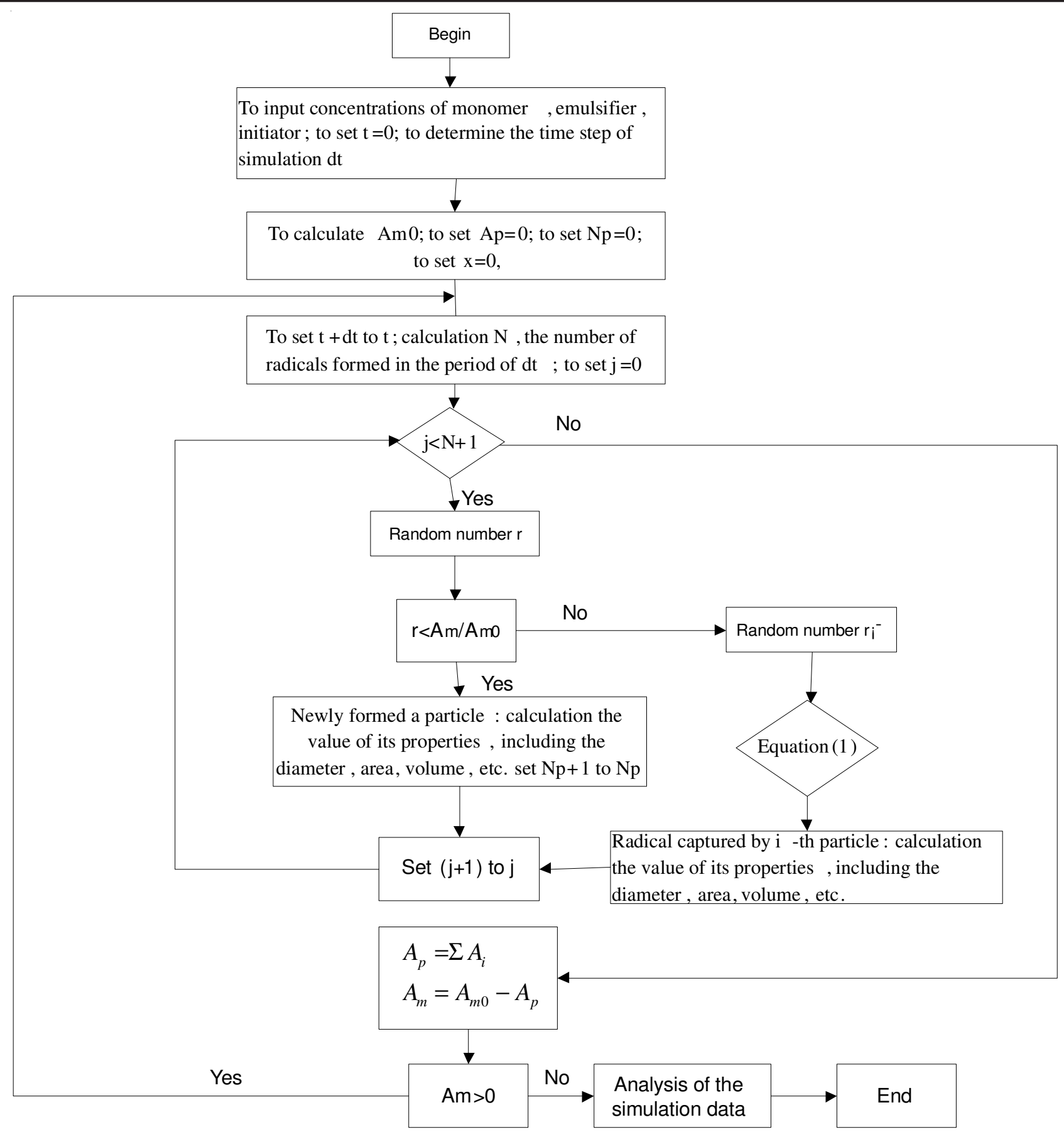

Fig. 1. Simulation flowchart of nucleation stage of emulsion polymerization for styrene butadiene rubber

1) Micelles use up all the emulsifier molecules before the polymerization. The emulsifier molecules dissolved in water and distributed on the surface of monomer droplets are neglected.

2) Emulsifier molecules are only distributed between micelles and the surface of latex particles after polymerization starts.

3) The area covered by a molecule of emulsifier is constant that does not change whether the molecule is in a micelle or on the surface of a latex particle.

4) In the nucleation stage, the volumetric ratio of polymer to monomer in latex particles is constant.

5) All free radicals formed in the aqueous phase are captured by micelles and particles. The capability for a micelle or a particle to capture the radicals is directly proportional to its surface.
6) Volumetric effects and gel effects are ignored.

Simulation: The emulsion polymerization was carried out on computer, assuming in a mini-reactor, whose volume contains about $10^{-18} \mathrm{~m}^{3}$ of water. So with the help of powerful personal computer, the decomposition of initiators, formation of radicals, entries of radicals to micelles and particles, growth of single particle, progress of monomer conversion and other processes in the emulsion polymerization can be simulated and recorded in details.

The detailed simulation procedure is shown in Fig. 1. The Monte Carlo method was involved in the simulation of the polymerization. When free radicals were formed in aqueous phase, they would be all captured by micelles at the beginning of the polymerization; but they would be partitioned between 
micelles and the latex particles after the polymerization started. The partition of free radicals between the micelles and latex particles was simulated by random numbers. The random number $r$ is of double type between 0 and 1.0. If $r \leq A_{m} / A_{m 0}$, the newly formed free radicals would be captured by micelles. Else, latex particles would capture the free radicals.

In the later case, every newly formed free radical would be captured by latex particles with different diameters; and it was simulated by random number, too. The latex particles were numbered and sorted by their diameters from small to large. Assume that there are $\mathrm{n}$ latex particles in the reactor and the surface area of the ith particle is $A_{i}$. The random number $r^{\prime}$ was used to determine which particle would capture the free radical. If $r$ ' meets the following eqn. (1), the free radical would be captured by ith particle.

$$
\sum_{\mathrm{j}=1}^{\mathrm{i}} \mathrm{A}_{\mathrm{j}} / \sum_{\mathrm{j}=1}^{\mathrm{i}} \mathrm{A}_{\mathrm{j}} \leq \mathrm{r}^{\prime}<\sum_{\mathrm{j}=1}^{\mathrm{i}+1} \mathrm{~A}_{\mathrm{j}} / \sum_{\mathrm{j}=1}^{\mathrm{n}} \mathrm{A}_{\mathrm{j}}
$$

Emulsion polymerization: The recipe for styrene/ butadiene polymerization used in this study is presented in Table-1 which was from the industrial recipe. Emulsion polymerization was carried out at $5-9^{\circ} \mathrm{C}$ in a $1.4 \mathrm{~L}$ polymerization autoclave by Yantai Songling Chemical Equipment Co. Ltd. For a emulsion copolymerization, the deionized water, surfactant, electrolyte solution, activator solution, modifier was first charged into the autoclave in turn, the autoclave was purged with nitrogen to remove the air for 3 times, the temperature of autoclave was controlled at $-6{ }^{\circ} \mathrm{C}$ and then the pressure cylinder containing the butadiene monomer was connected to the autoclave, the mass amount of butadiene was measured by the mass flow meter. Then, the temperature of the autoclave was increased to about $5^{\circ} \mathrm{C}$, the stirring speed was controlled at $80 \mathrm{rpm}$, the oxidant and the sodium hydrosulfite were charged into the autoclave. Then the emulsion polymerization happens. Samples of latex for various analyses were periodically withdrawn from the autoclave. These samples were short-stopped immediately with shortstop solution and placed in an ice bath. Latex was coagulated by addition of salt $(10 \%, \mathrm{NaCl})$ and acid $(3.4 \%$, $\mathrm{H}_{2} \mathrm{SO}_{4}$ ), soap-converted and resulted in rubber crumb by washing and drying.

\begin{tabular}{cc} 
TABLE-1 \\
CONCENTRATION OF COMPONENTS IN \\
THE POLYMERIZATION \\
\hline Components & Concentration $(\mathrm{mol} / \mathrm{L})$ \\
\hline Emulsifier & 0.0267 \\
Electrolyte & 0.0046 \\
SHS & 0.0002 \\
Activator & 0.0094 \\
Modifier & 0.0008 \\
Oxidant & 0.0094 \\
\hline
\end{tabular}

Total solid content in latex was measured according to SH/T 1154-1999. The viscosity of latex was measured on a NDJ-79 rotational viscometer by Shanghai Changji geological Instrument Co. Ltd.

The monomer conversion of polymerization $(\mathrm{X}, \%)$ was calculated as follows.

$$
X=295.619 \times \text { TSC-5.616 }
$$

The gel content was determined on the G2 glass sand funnel filter with toluene as solvent by weight increase method. The Mooney viscosity $\left(\mathrm{ML}_{1+4}^{100^{\circ} \mathrm{C}}\right)$ of rubber crumb was characterized on a JC-2000G mooney viscosity tester by Jiangdu jingcheng test intruments factory according to GB/T 12322000. The combining styrene content (CS, \%) of rubber crumb was identified on a UV1900 ultraviolet spectrophotometer by Shanghai Asia-reserch Electric Scientific Co. Ltd., according to GB/T 13646-92. The raw and vulcanizate properties of rubber crumb were measured according to GB/T 8655-2006 and GB/T 8656-2002.

\section{RESULTS AND DISCUSSION}

Nucleation stage simulation in emulsion polymerization for styrene butadiene rubber: The influences of initial emulsifier and initiator concentration on the monomer conversion in emulsion polymerization for styrene butadiene rubber were simulated (Fig. 2).
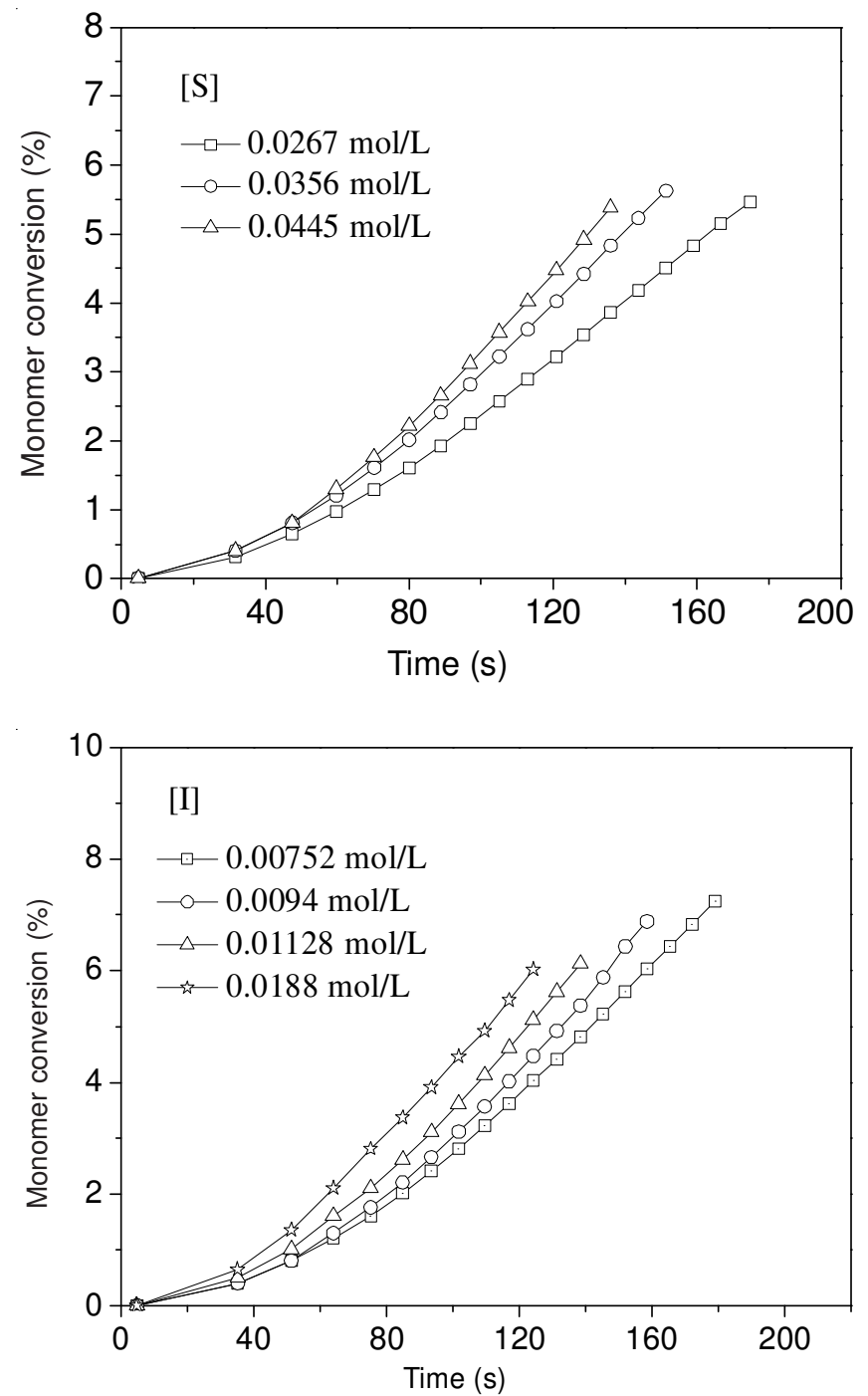

Fig. 2. Influences of initial emulsifier and initiator concentration on the monomer conversion in emulsion polymerization for SBR

The monomer conversion will become higher in the same reaction time. Fig. 2 also shows that with the increase of the 
initiator concentration, the nucleation stage will be finished more quickly, but with the increase of the emulsifier concentration, the nucleation stage will last much longer.

The particle numbers under different polymerizations are listed in Table-2. In Table-2, [I] and [S] are the concentration of initiator ( $\mathrm{mol} / \mathrm{L}$ water) and emulsifier (mol/L water), respectively. Much more results can be listed if needed. With the statistical analysis of the data, the equation can be fitted as:

$$
\mathrm{N}_{\mathrm{p}}=\mathrm{k}[\mathrm{S}]^{0.6062}[\mathrm{I}]^{0.4055}
$$

It is found that the equation of $\mathrm{N}_{\mathrm{p}}$ versus [S] and [I] accords with the Smith-Ewart equation. In addition, it is obvious that the results listed in Table-1 are not unique because the random method has been adopted in the simulation. When the polymerization is simulated at different time or with different computers, the results as well as all other data cited below such as particle diameters, surface areas, etc., are not the same but they are close to each other and in a statistical view they are equal.

\begin{tabular}{ccc}
\hline \multicolumn{3}{c}{ TABLE-2 } \\
NUMBER OF LATEX PARTICLES BY DIFFERENT \\
REACTION CONDITIONS \\
\hline$[\mathrm{S}] \mathrm{mol} / \mathrm{L}$ & {$[\mathrm{I}] \mathrm{mol} / \mathrm{L}$} & $\mathrm{Np}$ \\
\hline 0.0445 & 0.0094 & 339 \\
0.0445 & 0.01128 & 370 \\
0.0445 & 0.0188 & 432 \\
0.0445 & 0.00752 & 286 \\
0.0445 & 0.00564 & 256 \\
0.0356 & 0.0094 & 293 \\
0.0267 & 0.0094 & 241 \\
0.0178 & 0.0094 & 185 \\
0.0333 & 0.01245 & 300 \\
0.0333 & 0.03245 & 439 \\
0.0222 & 0.01287 & 250 \\
\hline
\end{tabular}

It is found that the more radicals or micelles in latex particles were formed by increasing the amount of emulsifier and initiator concentration. The monomer conversion can be increased at the same emulsion polymerization time and the more latex particles and more polymer chain was formed. Thus, the product which produced by increased monomer conversion can be similarity with the product which produced by the original industrial recipe.

The original industrial recipe was used for the monomer conversion $62 \%$ in emulsion polymerization for styrene butadiene rubber. And the product was satisfied with the superior product standard. The monomer conversion in emulsion polymerization for styrene butadiene rubber will be increased to $70 \%$. According to the eqn. (2), the eqn. (3) can be obtained as followed.

$$
\log \left(\frac{\mathrm{N}_{\mathrm{p} 1}}{\mathrm{~N}_{\mathrm{p} 2}}\right)=0.6062 \times \log \left(\frac{\left[\mathrm{S}_{1}\right]}{\left[\mathrm{S}_{2}\right]}\right)+0.4055 \times \log \left(\frac{\left[\mathrm{I}_{1}\right]}{\left[\mathrm{I}_{2}\right]}\right)
$$

$\mathrm{N}_{\mathrm{p}}$, [S], [I] denoted the number of micelles, the emulsifier concentration and initiator concentration, respectively. Subscript 1 represents the emulsion polymerization parameters in the monomer conversion of $62 \%$ and subscript 2 represents the emulsion polymerization parameters in the monomer conversion of $70 \%$.
If the emulsifier concentration unchanged, $\left[\mathrm{I}_{2}\right] /\left[\mathrm{I}_{1}\right]=$ 1.3489 was obtained. If the initiator concentration unchanged, $\left[\mathrm{S}_{2}\right] /\left[\mathrm{S}_{1}\right]=1.2216$ was obtained. If the polymer chain proportional the monomer conversion with the initiator efficiency keeps same, $\left[\mathrm{I}_{2}\right] /\left[\mathrm{I}_{1}\right]=1.129$ was obtained.

$$
\left[\mathrm{S}_{2}\right] /\left[\mathrm{S}_{1}\right]=1.126
$$

The simulation results showed that the monomer conversion in emulsion polymerization increased from 62 to $70 \%$ in the premise of ensuring product quality, the emulsifier incremental amount between 12.6 and $22.16 \%$ and the initiator incremental amount between 12.9 and $34.89 \%$.

Experimental study on increasing monomer conversion in emulsion polymerization for styrene butadiene rubber: The emulsifier incremental amount between 0 and $20 \%$ and the initiator incremental amount between 0 and $40 \%$ was investigated in experiments refer to the simulation results. The effect of the amount of emulsifier and initiator on the polymerization time which the monomer conversion was $70 \%$ was shown in Fig. 3.

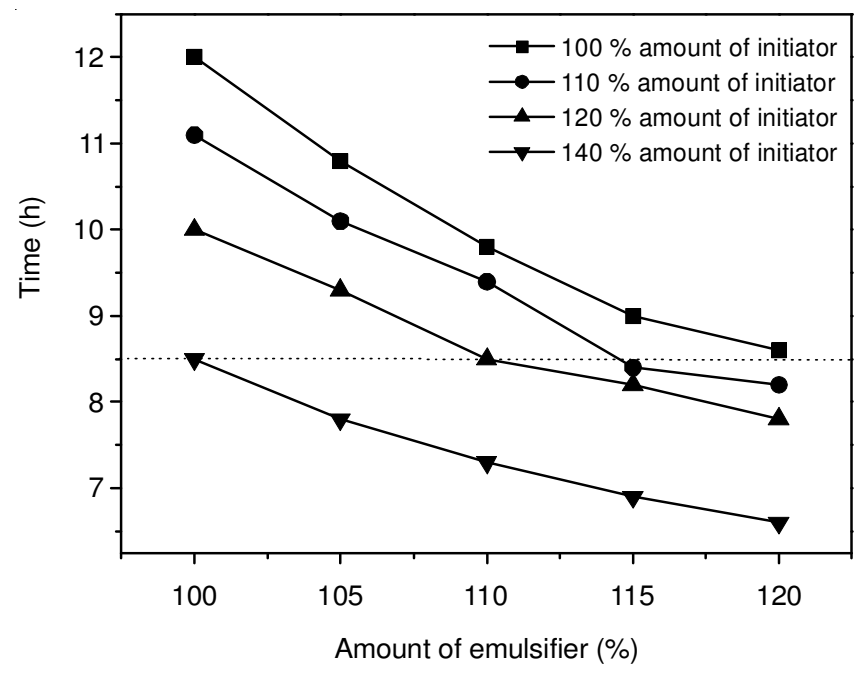

Fig. 3. Effect of the amount of emulsifier and initiator on the polymerization time

It can be seen that polymerization time which the monomer conversion reaches up to $70 \%$ was shorten by increasing the amount the initiator and emulsifier. When the incremental amount of emulsifier and initiator between 10 and $15 \%$, the polymerization time is $8.5 \mathrm{~h}$, which is equal with the emulsion polymerization of monomer conversion of $62 \%$. The original industrial recipe was adjusted as follows: the incremental amount of emulsifier was $10 \%$ and incremental amount of initiator was $15 \%$. The rubber crumb with monomer conversion of $70 \%$ according to the adjusted recipe was synthesized.

The raw and vulcanizate properties of rubber crumb with monomer conversion of $62 \%$ by original industrial recipe, rubber crumb with monomer conversion of $70 \%$ by adjusting recipe, the results and corresponding product standard were shown in Table-3. It can be seen that the raw and vulcanizate properties of rubber crumb high monomer conversion of $70 \%$ by adjusting recipe were satisfied to meet the requirements of the superior product standard of styrene butadiene rubber. 


\begin{tabular}{|c|c|c|c|}
\hline \multicolumn{4}{|c|}{$\begin{array}{c}\text { TABLE-3 } \\
\text { RAW RUBBER AND VULCANIZATE PROPERTIES OF } \\
\text { RUBBER CRUMB WITH DIFFERENT CONVERSION } \\
\text { AND CORRESPONDING PRODUCT STANDARD }\end{array}$} \\
\hline \multirow[t]{2}{*}{ Properties } & \multicolumn{2}{|c|}{ Conversion (\%) } & \multirow{2}{*}{$\begin{array}{l}\text { Superior produc } \\
\text { standard }\end{array}$} \\
\hline & 62 & 70 & \\
\hline \multicolumn{4}{|l|}{ Mooney viscosity } \\
\hline Raw rubber & 56 & 49 & $46-58$ \\
\hline Vulcanizate & 80 & 75 & $\leq 85$ \\
\hline Tensile strength (MPa) & 25.7 & 27.90 & $\geq 22.1$ \\
\hline \multicolumn{4}{|l|}{ Modulus at $300 \%(\mathrm{MPa})$} \\
\hline $25 \mathrm{~min}$ & 9.33 & 10.18 & 7.1-11.6 \\
\hline $35 \min$ & 14.66 & 15.17 & $11.3-15.8$ \\
\hline $50 \mathrm{~min}$ & 16.37 & 17.44 & $13.2-17.7$ \\
\hline Elongation at break $(\%)$ & 527.5 & 522.2 & $\geq 480$ \\
\hline
\end{tabular}

\section{Conclusion}

The nucleation stage in emulsion polymerization for styrene-butadiene-rubber (styrene butadiene rubber) was established by classic theory and the influences of emulsifier and initiator concentration on the reaction were simulated by Monte Carlo method. The results showed that the relationship of the number of latex particle $\left[\mathrm{N}_{\mathrm{p}}\right]$, emulsifier concentration $[\mathrm{S}]$ and initiator concentration $[\mathrm{I}]$ is to $\mathrm{N}_{\mathrm{p}}=\mathrm{k}[\mathrm{S}]^{0.6062}[\mathrm{I}]^{0.4055}$, what agrees well with the Smith-Ewart theory.

The simulation results showed that, the monomer conversion in emulsion polymerization increased from $62 \%$ to $70 \%$ in the premise of ensuring product quality, the emulsifier incremental amount between 12.6 and $22.16 \%$ and the initiator incremental amount between 12.9 and $34.89 \%$.

The experiments were investigated on increasing monomer conversion by emulsifier and emulsifier incremental amount in emulsion polymerization for styrene butadiene rubber according to the simulation results. The original industrial recipe was adjusted as follows: the incremental amount of emulsifier was $10 \%$ and incremental amount of initiator was $15 \%$. The raw and vulcanizate properties of rubber crumb high monomer conversion of $70 \%$ by adjusting recipe and monomer conversion of $62 \%$ by original recipe were all satisfied to meet the requirements of the superior product standard of styrene butadiene rubber. The experimental results verify the simulation results.

\section{REFERENCES}

1. D.H. Liu, G.B. Gong and J.P. Liu, Emulsion Polymerization for StyreneButadiene Rubber, China Petrochemical Press, Beijing, pp. 1-30 (2011).

2. J. Zhang, A.J. Morris and E.B. Martin, Chem. Eng. J., 69, 135 (1998).

3. C.D. Immanuel and F.J. Doyle, Chem. Eng. Sci., 58, 3681 (2003).

4. F.L.M. Marin, L.M.F. Lona, M.R.W. Maciel and R. Maciel, J. Appl. Polym. Sci., 102, 6037 (2006).

5. S.L. Lu, Y. Song, L. Zhang, S.K. Dong, Y.R. Li, J. Sui, G.Y. Zhang and J. Wang, China Synth. Rubb. Ind., 29, 245 (2006).

6. L.X. Song, China Elastomerics, 13, 20 (2003).

7. Y.J. Jin, B.X. Shen, J. Sui, M.H. Cui, L. Yang and J.G. Zhao, China Elastomerics, 21, 44 (2011).

8. L.J. Zhu and K.A. Narh, J. Polym. Sci., Part B: Polym. Phys., 42, 2391 (2004).

9. D.G. Martinez, A. Olivieri and C.E. Boschetti, Comput. Chem. Eng., 33, 850 (2009).

10. G. Padmavathi, M.G. Mandan and S.P. Mitra, Comput. Chem. Eng., 29, 1677 (2005).

11. A. Kalani and P.D. Christofides, Comput. Chem. Eng., 26, 1153 (2002).

12. D.G. Martinez and C.E. Boschetti, J. Appl. Polym. Sci., 124, 3468 (2012). 